\title{
Aprendizagem Baseada em Problemas aplicada à Programação de Computadores: Um Mapeamento Sistemático
}

Marcos da Conceição Coelho, Instituto Federal de Educação Ciência e Tecnologia do

Sertão Pernambucano Campus Petrolina, marcos.lcomputacao@gmail.com Albertina Marília Alves Guedes, Instituto Federal de Educação Ciência e Tecnologia do Sertão Pernambucano Campus Petrolina, albertina.guedes@ifsertao-pe.edu.br

Resumo: No presente artigo são apresentados os resultados de um mapeamento sistemático que traz uma discussão e reflexão sobre Aprendizagem Baseada em Problema (ABP) como uma Metodologia Ativa de ensino que pode elevar a capacidade de aprendizagem na disciplina de Programação. Esse estudo teve como principal objetivo identificar as contribuições e desafios da ABP no ensino de Programação. Foi utilizado uma string de busca em 10 mecanismos de busca, onde foram encontrados 316 estudos e selecionados 22 estudos primários (EPs) que foram utilizados para responder às questões da pesquisa. A partir dos estudos primários foram encontradas 35 contribuições e 30 desafios/dificuldades no ensino de Programação utilizando a $A B P$. Os resultados obtidos destacam que a maior contribuição da ABP na Programação é promover a habilidade de resolução de problemas. Além disso destacam-se a autonomia, trabalho em equipe e motivação. Por outro lado, os maiores desafios encontrados foram com relação a capacitação dos atores (professores e alunos) no processo da ABP e a exigência de maior esforço tanto dos professores quanto dos alunos. Com a realização desta pesquisa pode-se concluir que o número de estudos publicados sobre ABP na Programação tem crescido nos últimos anos e que a maior parte dos estudos primários mapeados eram voltados para alunos do ensino superior.

Palavras-chave: Aprendizagem Baseada em Problemas, Programação, Metodologias Ativas, Mapeamento Sistemático.

\section{Problem-Based Learning Applied to Computer Programming: Systematic Mapping}

\begin{abstract}
This article presents the results of a systematic mapping that brings a discussion and reflection on Problem-Based Learning (PBL) as an Active Teaching Methodology that can increase learning capacity in the Programming discipline. The main objective of this study was to identify the contributions and challenges of PBL in programming teaching. A search sequence was used in 10 search engines, where 316 studies were found and 22 primary studies (EPS) were selected that were used to answer the research questions. From the primary studies, 35 contributions and 30 challenges/difficulties in programming teaching were found using PBL. The results obtained highlight that the greatest contribution of PBL in programming is to promote the problem solving skill. In addition, autonomy, teamwork and motivation stand out. On the other hand, the greatest challenges encountered were in relation to the training of actors in the PBL process and the demand for greater effort of both teachers and students. With this research, it can be concluded that the number of studies published on PBL in programming has grown in recent years and that most of the mapped primary studies were aimed at higher education students.
\end{abstract}


Key-words: Problem-Based Learning, Programming, Active Methodologies, Systematic Mapping.

\section{Introdução}

Atualmente a presença do uso de instrumentos tecnológicos tem crescido progressivamente e, muitas vezes, está ligada a diversas áreas do conhecimento. Os estudantes que ingressam em cursos de ensino superior na área de informática e/ou tecnologias as quais podem estimular habilidades computacionais mais avançadas geralmente se deparam com dificuldades em assimilar e aprender os conteúdos que envolvem tais habilidades relacionadas aos conhecimentos tecnológicos principalmente porque a maioria das disciplinas iniciais diz respeito a Programação de computadores (MOREIRA et. al., 2018).

Na concepção de Moreira et. al. (2018) existem vários fatores que influenciam nas dificuldades no processo de ensino-aprendizagem de Programação as quais Raabe e Silva (2005) identificam como sendo: (1) problemas de natureza didática; (2) problemas de natureza cognitiva e; (3) problemas de natureza afetiva. Concernente os problemas de natureza didática dizem respeito a grande quantidade de alunos em sala de aula; dificuldade do professor compreender a lógica do aluno; diferença de experiência e ritmo de aprendizagem entre os alunos; ambiente de realização das provas; pouco uso dos monitores da disciplina; ausência de bons materiais e; alunos desorientados a escolha do curso. Os problemas de natureza cognitiva dizem respeito a alunos sem perfil para solução de problemas; alunos sem base operatório-formal e; conteúdo sem proximidade com o conteúdo escolar. Já os problemas de natureza afetiva são: ocasionais e constantes. Com isso, percebe-se a diversidade de problemas e a necessidade de identificação de metodologias ativas de ensino que facilitem o processo de aprendizagem do aluno, de forma que considere as diferenças empíricas e o tempo de aprendizagem de cada um, explorando aspectos concretos na Programação de computadores.

A partir deste contexto, Dias e Chaga (2017, p. 38) apresentam que uma possibilidade que pode contribuir para minimizar as dificuldades concernentes à aprendizagem de computação pode ser o uso de metodologias ativas por parte dos professores. Esse tipo de metodologia valorizam o processo de ensino e aprendizagem com foco no desenvolvimento da autonomia do aprendiz, desenvolvendo habilidades de identificar, descrever e solucionar problemas, propondo soluções práticas, a partir da realização de atividades em equipes ou com base na construção de projetos, dentre outras possibilidades, tais como, a Aprendizagem Baseada em Problemas (ABP).

Conforme descrito por Ribeiro (2005), a ABP é essencialmente um método de instrução caracterizado pelo uso de problemas da vida real para estimular o desenvolvimento de pensamento crítico e habilidades de solução de problemas e a aprendizagem de conceitos fundamentais da área do conhecimento em questão. Neste contexto, a ABP proporciona ao aluno uma aprendizagem autônoma, bem como a contextualização e aplicação de situações reais na Programação de Computadores. Portanto, o principal objetivo deste estudo é identificar as contribuições e desafios da Aprendizagem baseada em Problemas no ensino de Programação.

\section{Materiais e Métodos}

O procedimento de coleta de dados deste trabalho diz respeito a um mapeamento sistemático que buscou identificar as contribuições e desafios da Aprendizagem Baseada em Problemas no ensino de Programação. Para direcionar as atividades de 
execução do MS, foram utilizadas as recomendações de Kitchenham e Charters (2007), que dividem o mapeamento sistemático em três fases distintas: planejamento, condução e publicação dos resultados.

$\mathrm{Na}$ etapa de planejamento foram identificadas as necessidades da pesquisa, ou seja, a motivação para a execução de um mapeamento sistemático. O trabalho tem como objetivo principal apresentar um mapeamento sistemático sobre as contribuições e desafios da ABP no ensino de Programação. A partir desse objetivo foi definido o protocolo do MS, que consiste nas questões de pesquisa, strings de busca e as fontes de pesquisa, bem como, os critérios de seleção e qualidade. Em seguida, foi realizada a avaliação do protocolo.

Após o protocolo ter sido definido foi realizada a condução do Mapeamento Sistemático. Inicialmente foram conduzidas às estratégias de busca para identificar estudos. A partir dos critérios de seleção e qualidade, serão selecionados os estudos primários. Posteriormente, os dados serão extraídos e sintetizados. A última fase consistiu na descrição e divulgação dos resultados obtidos no mapeamento sistemático.

\subsection{Questões de Pesquisa}

Este estudo teve por objetivo identificar as contribuições e desafios da Aprendizagem Baseada em Problemas no ensino de Programação. Assim, a pesquisa partiu das seguintes questões: QP1) Quais as vantagens e benefícios na aplicação da ABP no ensino de Programação? QP2) Quais são os principais desafios e dificuldades na utilização da ABP? QP3) Como tem sido a evolução do número de estudos sobre ABP? QP4) Quais os níveis de ensino onde a ABP é mais utilizada, e o público alvo mais contemplado?

A fim de responder às questões da pesquisa, foi utilizada a estratégia de busca abaixo (ver Figura 1) para determinar os termos de busca.

Figura 1: Estratégia para realização das buscas

Identificar as palavras-chave que norteiam as questões de pesquisa
Traduzir os termos para lingua inglesa
Identificar os sinônimos dos termos escolhidos
$\begin{array}{r}\text { Gerar a string de busca de acordo com as palavras-chave e respectivos sinônimos. Para } \\ \text { tanto, utilizar os operadores lógicos 'OR'e 'AND'. }\end{array}$

Fonte: Adaptado de Kitchenham e Charters (2007)

De acordo com as estratégias definidas acima, as palavras-chave e respectivos sinônimos traduzidos para a língua inglesa utilizados na busca foram:

- Aprendizagem baseada em problemas, ABP. Problem-Based Learning, PBL.

- Programação, Programação de computadores. Programming, computer programming.

Com as palavras-chave encontradas, foram geradas as strings de busca, como mostra o Quadro 1 abaixo:

Quadro 1: Strings de busca utilizada no mapeamento sistemático

\begin{tabular}{|l|}
\hline \multicolumn{1}{|c|}{ Strings de busca } \\
\hline$(($ "problem-based learning" OR "PBL") AND ("programming" OR "computer programming")) \\
\hline $\begin{array}{l}\text { (("aprendizagem baseada em problemas" OR "ABP" OR "PBL") AND ("Programação" OR "Programação de } \\
\text { computadores")) }\end{array}$ \\
\hline
\end{tabular}

Fonte: elaborado pelo autor (2019). 
A string em língua inglesa foi utilizada nos mecanismos de busca: IEEEXplore, ACM, SciELO, Microsoft Academic e BDTD. A string em português foi utilizada nos demais repositórios: RENOTE, WEI, WIE, SBIE e CBIE.

\subsection{Critérios de inclusão e exclusão}

Os critérios de inclusão e exclusão utilizados para a seleção dos estudo primários estão descritos no Quadro 2 abaixo:

Quadro 2: Critérios de inclusão e exclusão dos estudos

\begin{tabular}{|l|l|}
\hline Foram incluídos os estudos: & Foram excluídos os estudos: \\
\hline Disponíveis exclusivamente em meio eletrônico; & $\begin{array}{l}\text { Repetidos: se o trabalho for reproduzido novamente em } \\
\text { fontes de busca diferentes; }\end{array}$ \\
\hline $\begin{array}{l}\text { Disponíveis em repositórios web de relevânciala } \\
\text { científico-acadêmico; }\end{array}$ & $\begin{array}{l}\text { Duplicados: trabalhos com estudos semelhantes. Neste } \\
\text { caso foi apenas o trabalho mais recente ou com } \\
\text { informações mais completas; }\end{array}$ \\
\hline $\begin{array}{l}\text { Verificar, a partir de testes, se o mecanismo escolhido } \\
\text { retorna estudos com as palavras-chaves acima citadas; }\end{array}$ & Irrelevantes para o objetivo da pesquisa; \\
\hline $\begin{array}{l}\text { Os trabalhos que demonstraram embasamento científico } \\
\text { que comprovaram os seus resultados; }\end{array}$ & Com textos, conteúdo ou resultados incompletos. \\
\hline
\end{tabular}

Fonte: elaborado pelo autor (2019).

\section{Resultados e Discussão}

Esta etapa apresenta os resultados obtidos através do Mapeamento Sistemático. A busca resultou em 316 artigos: 106 do IEEEXplore, 135 do ACM, 4 do SciElo, 25 DO Microsoft Academic, 8 do BDTD, 5 da RENOTE, 6 do WEI, 6 do WIE, 15 do SBIE E 6 do CBIE. Após a primeira seleção (leitura dos títulos e palavras-chaves) dos trabalhos, resultaram 48 estudos potencialmente relevantes para a pesquisa. Na segunda seleção foi realizada a leitura da introdução e conclusão dos estudos. Depois, foram excluídos os estudos a partir dos critérios definidos. A partir dos estudos resultantes dessa seleção houve a leitura integral dos estudos incluídos. Nesta etapa, 26 estudos foram descartados e 22 foram incluídos, que foram considerados como estudos primários (EP) e foram fichados no Formulário de Aprovação de Trabalhos (https://docs.google.com/document/d/15pgU9jPf5by4PbvrWfrfj-z-ZpDo1aUsQGBq6t6 xh9w/edit?usp=sharing).

\subsection{Contribuições da aplicação da ABP no ensino de Programação}

Esta subseção apresenta a resposta a QP1 que visava investigar as vantagens e benefícios na aplicação da metodologia ABP no ensino de Programação de computadores. Tais contribuições foram relatadas nos estudos primários pelos respectivos autores. A maioria dos EPs apresentou pelo menos uma contribuição. $\mathrm{O}$ Quadro 3 abaixo apresenta as vantagens e benefícios mapeados, com o seu identificador e estudos primários dos quais foram extraídas.

Quadro 3: Vantagens e benefícios na aplicação da ABP no ensino de Programação

\begin{tabular}{|l|l|l|}
\hline D-ID & TÓPICO & EPs \\
\hline C-01 & Desenvolvimento de diversas habilidades & $\begin{array}{l}\text { EP02, EP05, EP18, EP19, } \\
\text { EP20, EP21 }\end{array}$ \\
\hline C-02 & Resolução de problemas. Promove habilidades para resolver problemas. & $\begin{array}{l}\text { EP02, EP08, EP09, EP10, } \\
\text { EP12, EP15, EP16, EP18, } \\
\text { EP19, EP20, EP21, EP22 }\end{array}$ \\
\hline C-03 & $\begin{array}{l}\text { Trabalho em Equipe. Torna os alunos responsáveis e preparados para trabalhar } \\
\text { com os colegas. Desenvolver habilidades como a capacidade de trabalhar como } \\
\text { um membro da equipe. }\end{array}$ & $\begin{array}{l}\text { EP02, EP03, EP04, EP06, } \\
\text { EP08, EP11, EP13, EP17, } \\
\text { EP19, EP21 }\end{array}$ \\
\hline C-04 & $\begin{array}{l}\text { Autonomia na Aprendizagem. Incentiva o autoaprendizado, ou seja, } \\
\text { aprendizagem autônoma }\end{array}$ & $\begin{array}{l}\text { EP02, EP04, EP06, EP07, } \\
\text { EP08, EP10, EP14, EP20, } \\
\text { EP21, EP22 }\end{array}$ \\
\hline
\end{tabular}




\begin{tabular}{|c|c|c|}
\hline $\mathrm{C}-05$ & Aperfeiçoamento das habilidades de comunicação & $\begin{array}{l}\text { EP02, EP06, EP08, EP17, } \\
\text { EP19, EP21 }\end{array}$ \\
\hline $\mathrm{C}-06$ & Motivação e/ou estímulo para a aprendizagem. & $\begin{array}{l}\text { EP03, ЕP04, ЕР06, ЕР07, } \\
\text { EP08, EP11, ЕP13, ЕP17, } \\
\text { EP20, EP22 }\end{array}$ \\
\hline $\mathrm{C}-07$ & $\begin{array}{l}\text { Aplicabilidade das teorias de forma direcionada, contextualizada e } \\
\text { produtiva, tornando o aluno participante do processo. }\end{array}$ & EP03 \\
\hline $\mathrm{C}-08$ & Organizar e otimizar processos & EP03 \\
\hline $\mathrm{C}-09$ & Compreender o porquê da aprendizagem de cada conteúdo e sua aplicação & EP03 \\
\hline $\mathrm{C}-10$ & $\begin{array}{l}\text { Aumento do senso de responsabilidade dos alunos. Os alunos assumem mais } \\
\text { responsabilidade para seus processos de aprendizagem pessoal em salas de aula } \\
\text { aplicando o modelo de ABP. }\end{array}$ & $\begin{array}{l}\text { EP03, EP10, EP11, EP20, } \\
\text { EP22 }\end{array}$ \\
\hline $\mathrm{C}-11$ & Estímulo à leitura e às discussões & EP03, EP20 \\
\hline $\mathrm{C}-12$ & $\begin{array}{l}\text { Incentivo à investigação mais detalhada e aprofundada do problema } \\
\text { apresentado. Os alunos podem alcançar o conhecimento científico escondido por } \\
\text { trás do problema }\end{array}$ & EP04, EP08, ЕР20 \\
\hline $\mathrm{C}-13$ & $\begin{array}{l}\text { Aprimorar as habilidades de pesquisa. Buscar e selecionar melhor o } \\
\text { conhecimento de que necessitam. }\end{array}$ & EP04, EP05, EP12, EP20 \\
\hline $\mathrm{C}-14$ & $\begin{array}{l}\text { Compartilhar ideias com os colegas para ampliar sua visão para resolver } \\
\text { problemas. }\end{array}$ & EP04, EP08 \\
\hline $\mathrm{C}-15$ & Curiosidade para buscar novos conhecimentos & EP04 \\
\hline $\mathrm{C}-16$ & Uso do conhecimento prévio & EP04, EP08 \\
\hline $\mathrm{C}-17$ & $\begin{array}{l}\text { Dá ao aluno um papel importante no aprendizado. O modelo PBL transforma } \\
\text { os alunos de passivos receptores de informação em indivíduos ativos, } \\
\text { auto-aprendizes e que resolvem problemas. }\end{array}$ & EP04, EP10, EP20 \\
\hline $\mathrm{C}-18$ & Construir uma aprendizagem significativa & EP05, ЕР08 \\
\hline $\mathrm{C}-19$ & Promover a colaboração & EP05, EP08, EP10, EP22 \\
\hline $\mathrm{C}-20$ & Pensamento crítico & EP05, EP09, EP12 \\
\hline $\mathrm{C}-21$ & Promove a proatividade dos alunos & EP06, ЕР20 \\
\hline $\mathrm{C}-22$ & Melhora a retenção de conteúdo & EP06, EP12 \\
\hline $\mathrm{C}-23$ & Estimula o raciocínio lógico & EP03, EP06, EP15 \\
\hline $\mathrm{C}-24$ & $\begin{array}{l}\text { Estimula os alunos a buscar o enquadramento teórico e prático para a solução } \\
\text { do problema }\end{array}$ & $\begin{array}{l}\text { EP07, EP11, EP15, EP18, } \\
\text { EP21 }\end{array}$ \\
\hline $\mathrm{C}-25$ & Facilita o desenvolvimento de um projeto & EP07, EP19 \\
\hline $\mathrm{C}-26$ & $\begin{array}{l}\text { Proporciona aos alunos um envolvimento e uma consciência real sobre os } \\
\text { problemas que eles têm que enfrentar na profissão, como trabalhar } \\
\text { eficientemente sob pressão e cumprir prazos, o que será crucial para a sua vida } \\
\text { profissional. }\end{array}$ & EP07, EP11 \\
\hline $\mathrm{C}-27$ & Aprender cooperativamente & EP07, EP08, EP19 \\
\hline $\mathrm{C}-28$ & $\begin{array}{l}\text { Melhora o desempenho acadêmico geral dos alunos na } \\
\text { Programação }\end{array}$ & EP08, ЕР09, ЕР12, ЕР16 \\
\hline $\mathrm{C}-29$ & $\begin{array}{l}\text { Habilidades técnicas. Possibilita a adaptação de técnicas profissionais para } \\
\text { serem utilizadas na ABP. }\end{array}$ & EP11, EP13, EP21 \\
\hline $\mathrm{C}-30$ & Promove a liderança & EP14, EP21 \\
\hline $\mathrm{C}-31$ & Promover a sua capacidade de pensamento computacional. & EP17 \\
\hline $\mathrm{C}-32$ & $\begin{array}{l}\text { Melhorar o desempenho dos professores na compreensão das disciplinas } \\
\text { relevantes }\end{array}$ & EP20 \\
\hline $\mathrm{C}-33$ & Melhorar a relacionamento entre professores e aluno & EP20 \\
\hline $\mathrm{C}-34$ & Promover a conversão do papel dos professores & EP20 \\
\hline $\mathrm{C}-35$ & $\begin{array}{l}\text { Melhora a capacidade de compreender o programa, de análise e desenho de } \\
\text { algoritmos e de escrever programas. }\end{array}$ & EP20 \\
\hline
\end{tabular}

Fonte: Elaborado pelo autor (2020)

O Quadro 3 acima apresenta 35 contribuições distintas sobre a aplicação da Aprendizagem baseada em problemas no ensino de Programação, distribuídas entre os 22 estudos primários mapeados. A contribuição de maior relevância foi promover a habilidade de resolução de problemas, citada em 12 estudos primários (54,5\%). Em seguida, mais três contribuições relevantes foram citadas em 10 estudos primários, cada uma.

A capacidade e promoção do trabalho em equipe foi exaltada em 10 estudos primários, um deles destacou que a $\mathrm{ABP}$ torna os alunos responsáveis e preparados para 
trabalhar com os colegas, outro EP diz que "a ABP ajuda a desenvolver habilidades como a capacidade de trabalhar como um membro da equipe".

Outra contribuição apresentada em 10 estudos primários, foi a autonomia na aprendizagem. Um dos EPs destaca que a ABP incentiva o autoaprendizado, ou seja, aprendizagem autônoma. Outro tópico relevante foi o destaque para a Motivação e/ou estímulo para a aprendizagem, 10 estudos deixam claro essa evidência.

Estas contribuições denotam a importância da aplicação da Aprendizagem baseada em problemas no ensino de Computação, principalmente para o desenvolvimento de habilidades de resolução de problemas, autonomia, trabalho em equipe e motivação.

\subsection{Desafios e dificuldades na aplicação da ABP}

Esta subseção apresenta a resposta a QP2 que visava investigar os principais desafios e dificuldades na utilização da ABP no ensino de Programação de computadores. Tais desafios/dificuldades foram apresentadas pelos respectivos autores dos estudos primários utilizados na pesquisa. Apesar de muitos EPs não apresentarem nenhum desafio/dificuldade, no total foram encontradas um número considerável de tópicos, que estão apresentados no Quadro 4 abaixo, acompanhados do seu identificador e estudos primários dos quais foram extraídos.

Quadro 4: Desafios e dificuldades na aplicação da ABP no ensino de Programação

\begin{tabular}{|c|c|c|}
\hline D-ID & TÓPICO & EPs \\
\hline $\mathrm{D}-01$ & $\begin{array}{l}\text { Qualidade dos problemas. O cuidado na elaboração dos problemas é relevante para a } \\
\text { motivação dos estudantes. }\end{array}$ & EP01, EP21, EP22 \\
\hline $\mathrm{D}-02$ & $\begin{array}{l}\text { O excesso de conceitos pode interferir no entendimento e no desenvolvimento da solução } \\
\text { do problema. }\end{array}$ & EP01, EP21 \\
\hline $\mathrm{D}-03$ & $\begin{array}{l}\text { A escolha do domínio do problema é relevante e deve introduzir fundamentos próximos } \\
\text { a realidade dos estudantes, sendo uma estratégia para tornar o problema mais atraente e } \\
\text { motivador. }\end{array}$ & EP01, EP21 \\
\hline $\mathrm{D}-04$ & $\begin{array}{l}\text { A complexidade do problema deve garantir que, com cooperação, os estudantes consigam } \\
\text { solucionar o problema. }\end{array}$ & EP01, EP10, EP21 \\
\hline $\mathrm{D}-05$ & A inserção de novidades é essencial para manter os níveis de motivação. & EP01 \\
\hline $\mathrm{D}-06$ & Falta de crença e conhecimento sobre a metodologia & EP02 \\
\hline $\mathrm{D}-07$ & Dificuldades nas disciplinas e na aplicação da metodologia. & EP02 \\
\hline $\mathrm{D}-08$ & Falta de tempo & EP02, EP22 \\
\hline $\mathrm{D}-09$ & $\begin{array}{l}\text { Incluir todos os alunos. Alguns estudantes podem se sentir perdidos ou excluídos nas } \\
\text { atividades. O problema de como dar aos alunos igualdade de oportunidades para práticas } \\
\text { em grupo nas aulas. }\end{array}$ & EP04, EP20 \\
\hline $\mathrm{D}-10$ & $\begin{array}{l}\text { Tornar os alunos independentes desde o início do curso, para enfrentar e investigar o } \\
\text { PROBLEMA que foi estabelecido. }\end{array}$ & EP07 \\
\hline $\mathrm{D}-11$ & $\begin{array}{l}\text { Obriga os alunos a trabalhar em grupo e pode criar dificuldades para aqueles que têm } \\
\text { dificuldades de adaptação a essa abordagem. }\end{array}$ & EP07 \\
\hline $\mathrm{D}-12$ & Avaliação. As avaliações individuais dos alunos são mais complexas na ABP & EP07, EP21 \\
\hline D-13 & $\begin{array}{l}\text { O sucesso do ABP ou de outros métodos depende muito da capacitação dos atores } \\
\text { (professores e alunos) no modelo. }\end{array}$ & \begin{tabular}{|l} 
EP07, EP08, EP13, \\
EP22
\end{tabular} \\
\hline D-14 & $\begin{array}{l}\text { Requer do professor um uso flexível do conhecimento, excelente lógica habilidade de } \\
\text { pensar, a habilidade de levantar questões e respostas, a capacidade de mobilizar o } \\
\text { interesse dos alunos e entusiasmo para discutir e a capacidade de organizar } \\
\text { ordenadamente o estudantes. }\end{array}$ & EP08 \\
\hline D-15 & $\begin{array}{l}\text { Os professores devem prestar atenção ao conhecimento acumulado de todos os aspectos, } \\
\text { melhorar sua própria capacidade e aumentar a qualidade do ensino através da aplicação } \\
\text { de aprendizagem. }\end{array}$ & EP08 \\
\hline D-16 & $\begin{array}{l}\text { Embora a ABP seja uma abordagem centrada no estudante e orientada a problemas, o } \\
\text { conteúdo e os cenários ainda são centrados no professor. }\end{array}$ & EP09 \\
\hline D-17 & $\begin{array}{l}\text { A construção de uma comunidade ABP abrangente requer determinação e } \\
\text { comprometimento de todos níveis - alunos, corpo docente e gerência - para que } \\
\text { funcione }\end{array}$ & EP09, EP18 \\
\hline D-18 & $\begin{array}{l}\text { As questões que a ABP pretende resolver são situações complexas que podem ser } \\
\text { experiência na vida diária. Estes problemas exigem habilidades de pensamento } \\
\text { algorítmico. }\end{array}$ & EP10 \\
\hline
\end{tabular}




\begin{tabular}{|l|l|l|}
\hline D-19 & $\begin{array}{l}\text { Alguns alunos têm dificuldade durante a ABP em termos de compreensão dos problemas } \\
\text { apresentados e } \\
\text { futuros com soluções. }\end{array}$ & EP10 \\
\hline D-20 & Exige um esforço maior tanto dos professores quanto dos alunos. & $\begin{array}{l}\text { EP11, EP13, EP20, } \\
\text { EP22 }\end{array}$ \\
\hline D-21 & Aumento da carga de trabalho de professores e alunos & EP11, EP20, EP22 \\
\hline D-22 & $\begin{array}{l}\text { Os professores devem constantemente melhorar a si mesmos e comunicar mais } \\
\text { frequentemente com professores de matérias relevantes. }\end{array}$ & EP08 \\
\hline D-23 & Cada aula deve ser projetada cuidadosamente & EP17 \\
\hline D-24 & $\begin{array}{l}\text { Transformar um processo de ensino conduzido pelo professor em um processo de } \\
\text { aprendizagem focado no aluno, que deve ser ativo na busca de conhecimento. }\end{array}$ & EP18, EP20, EP22 \\
\hline D-25 & Proporciona um ambiente produtivo para a experiência de aprendizagem & EP19 \\
\hline D-26 & Estimula a interação e o bem-estar emocional & EP19 \\
\hline D-27 & Contradição de tantos alunos, turmas grandes, menos horas e professores insuficientes. & EP20 \\
\hline D-28 & $\begin{array}{l}\text { Não há materiais de referência prontos de Aprendizagem do Modelo PBL, ele só pode ser } \\
\text { comprontrado pelos professores que, com base no material existente, combinado com }\end{array}$ & EP20 dos professores, e com base em muita coleta de informações. \\
\hline D-29 & $\begin{array}{l}\text { Liberar do modo tradicional de ensino. Especialmente no estudo inicial, os alunos não } \\
\text { estão acostumados com o novo modo de ensino e aprendizagem, todo o processo de } \\
\text { ensino é lento e difícil. }\end{array}$ & EP20, EP22 \\
\hline D-30 & $\begin{array}{l}\text { Requer que o aluno seja autodidata. Aqueles que não são autodidatas enfrentam } \\
\text { dificuldades neste novo contexto }\end{array}$ & EP21 \\
\hline
\end{tabular}

Fonte: Elaborado pelo autor (2020)

O Quadro 4 acima apresenta 30 desafios/dificuldades distintas sobre a aplicação da Aprendizagem baseada em problemas no ensino de Programação, distribuídas entre os 22 estudos primários mapeados. Os dois desafios considerados mais relevantes foram citados em 4 EPs distintos. O primeiro desafio (D-13) diz respeito à capacitação dos atores (professores e alunos) no processo da ABP, onde o sucesso desse método é muito dependente do nível desta capacitação. O segundo (D-20) diz respeito à exigência de maior esforço tanto dos professores quanto dos alunos.

Além desses, mais quatro desafios/dificuldades foram considerados relevantes e cada um deles foi citados em 3 EPs distintos. O terceiro (D-01) trata-se da preocupação com a qualidade dos problemas, pois esta é relevante para a motivação dos estudantes. No EP21 destaca-se que "um problema bem elaborado pode elevar os níveis motivacionais, enquanto um problema mal planejado pode ter resultados catastróficos. A qualidade dos problemas de ABP está entre os maiores desafios da abordagem." No EP22 os autores destacam que "um problema mal estruturado pode ser percebido pelos alunos como uma falta de conhecimento ou esforço por parte dos professores, e isso poderia ter um impacto negativo na avaliação de desempenho do ensino".

O quarto desafio (D-04) trata-se da complexidade do problema que deve garantir que, com cooperação, os estudantes consigam solucionar o problema, ou seja, o problema sugerido pelo professor deve está de acordo com o nível de conhecimento do aluno. O quinto desafio/dificuldade (D-21) é sobre o aumento da carga de trabalho de professores e alunos, que é maior no método de Aprendizagem baseada em problemas. O sexto (D-24) diz respeito à transformação do processo de ensino conduzido pelo professor em um processo de aprendizagem focado no aluno, que deve ser ativo na busca de conhecimento.

Os desafios e dificuldades apontadas pelos autores sugerem que o método ABP necessita ser conhecido pelo professor e pelo aluno, levando em conta fatores como o conhecimento prévio do aluno, e que os atores envolvidos no processo estejam comprometidos em usar mais tempo para buscar o conhecimento. Além disso, é necessário que os problemas sejam bem estruturados e condizentes com o nível dos alunos. 


\subsection{Evolução do número de estudos sobre ABP}

Esta subseção apresenta a resposta a QP3 que visava investigar a evolução do número de estudos sobre ABP no ensino de Programação de computadores. A busca pelos estudos primários considerou os 10 últimos anos de publicações, sendo estes estudos encontrados entre 2010 e 2019. Pode-se observar que nos últimos 2 anos o número de estudos é maior, e representa a maior parte dos estudos primários, o que denota a popularização da ABP atualmente. A disposição da quantidade de estudos por ano de publicação pode ser verificada no Gráfico 1 abaixo.

Gráfico 1: Número de Estudos Primários de 2010 a 2019

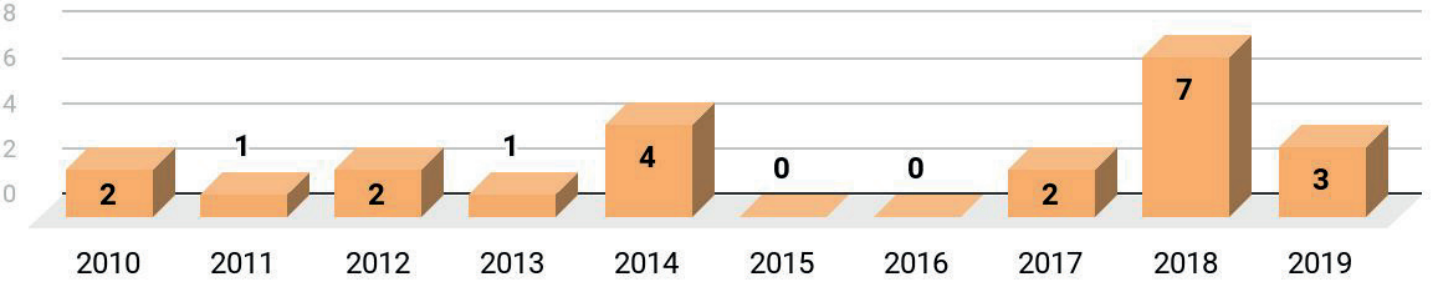

Fonte: Elaborado pelo autor (2020)

De acordo com o gráfico 1 acima, nos anos de 2015 e 2016 não houve nenhum estudo primário selecionado. Nos anos de 2011 e 2013 houve apenas 1 estudo por ano. Seguido por 2 trabalhos no ano de 2010, 2012 e 2017. O ano com o maior número de publicações entre os estudos primários foi em 2018, com 7 estudos. Esta observação sugere uma tendência recente no desenvolvimento de pesquisas sobre ABP, principalmente por ser uma temática difundida há poucos anos na área da Computação.

\subsection{Classificação dos EPs de acordo com o nível de ensino}

Esta subseção apresenta a resposta a QP4 que visava classificar os estudos primários utilizados neste mapeamento sistemático de acordo com o nível de ensino. As evidências encontradas concentram-se em Ensino Superior e Ensino Técnico, conforme apresentadas no Gráfico 2 abaixo.

\section{Gráfico 2: Estudos Primários quanto ao nível de ensino}

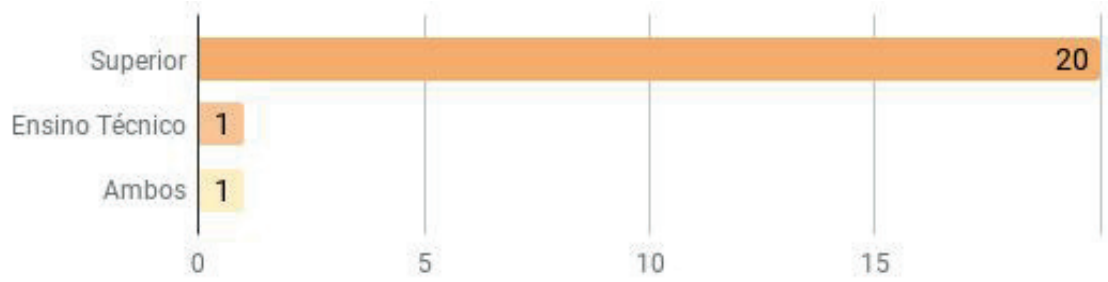

Fonte: Elaborado pelo autor (2020)

De acordo com os dados apresentados, pode-se observar que a maioria dos estudos retratam experiências voltadas somente para o ensino superior, com representação em 20 estudos primários, o qual corresponde a 90,9\% do total. Tais resultados sugerem que a Aprendizagem baseada em problemas aplicada ao ensino de Programação tem sido mais difundida no ensino superior. Percebe-se ainda que nenhum dos EPs apresenta trabalhos relacionados ao ensino básico, o que justifica-se pelo fato da Programação ser mais difundida na graduação e ensino técnico.

\subsection{Classificação dos EPs de acordo com o país de publicação}

Os estudos primários estão distribuídos em 12 países, dos quais o Brasil tem a maior representatividade, com 5 estudos selecionados. Em seguida, Estados Unidos que 
tiveram 4 estudos, e China, Canadá e Turquia, cada um com 2 estudos. Os outros países não representam um número significativo. O Gráfico 3 apresenta esta distribuição de publicações por países.

\section{Gráfico 3: Representatividade dos estudos por país}

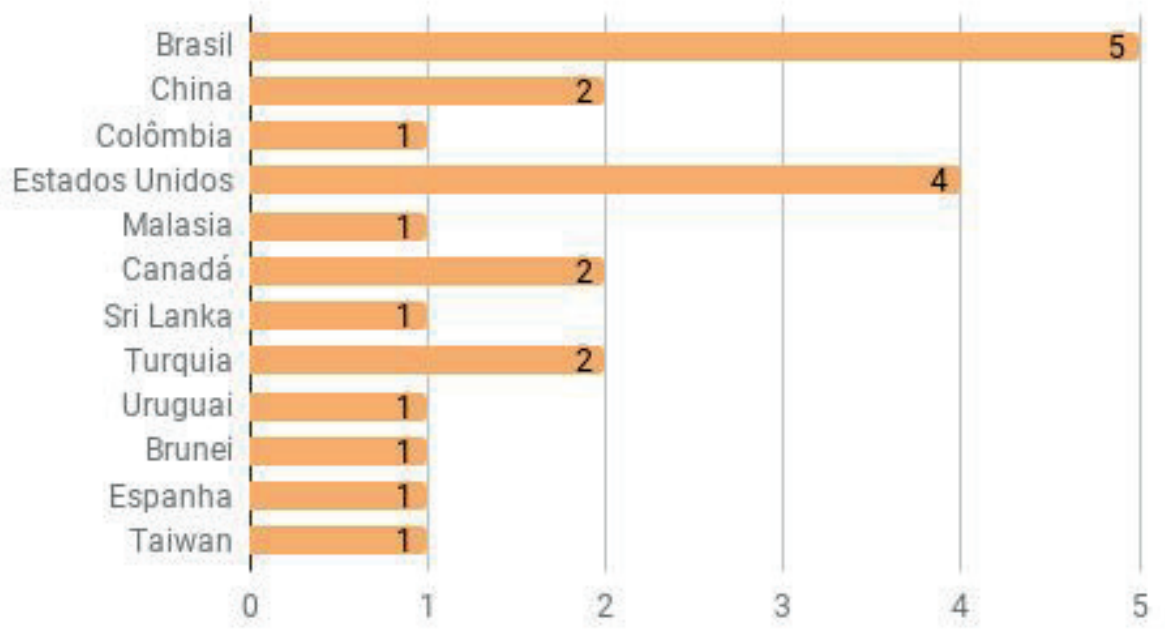

Fonte: Elaborado pelo autor (2020)

\section{Conclusão e Trabalhos futuros}

Este trabalho apresentou os resultados de um mapeamento sistemático da literatura, com o objetivo de identificar as contribuições e desafios da Aprendizagem baseada em Problemas no ensino de Programação. Foram desenvolvidas quatro questões de pesquisa para nortear o estudo e após a análise de 316 estudos, destes 22 estudos primários foram selecionados para responder às questões da pesquisa.

No que diz respeito a representatividade dos estudos por fontes de busca, o repositório ACM Library apresentou o maior número de estudos retornados $(42,7 \%)$, no entanto apenas 1 estudo foi relevante para a pesquisa, ou seja, sua representatividade no total dos estudos primários foi apenas de 4,5\%. Por outro lado, o repositório IEEExplore teve a maior número de estudos primários, com representatividade de $45,5 \%$ do total.

Com os resultados foi possível identificar 35 contribuições diferentes, distribuídas entre os 22 estudos primários. O número significativo de vantagens/benefícios permite inferir sobre a grande relevância da aplicação da ABP no ensino de Programação. A principal contribuição mapeada diz respeito à habilidade de resolução de problemas. Esta contribuição foi descrita em 54,5\% dos estudos primários selecionados.

Em relação aos desafios e as dificuldades na aplicação da ABP, a coleta de dados dos estudos primários permitiu observar que os principais desafios na aplicação da $\mathrm{ABP}$ são: 1) Capacitação dos atores (professores e alunos) no processo da $\mathrm{ABP}$ e; 2) A exigência de maior esforço tanto dos professores quanto dos alunos. Outros estudos destacaram a qualidade dos problemas, a complexidade destes, o aumento da carga de trabalho de professores e alunos, e a transformação do processo de ensino conduzido pelo professor em um processo de aprendizagem focado no aluno. Com isso, esses desafios/dificuldades são tópicos essenciais a serem considerados nas aplicações futuras de experiências utilizando a ABP.

Quanto à evolução do número de estudos envolvendo $\mathrm{ABP}$ no ensino de Programação, o ano de 2018 apresenta o maior número de publicações. Em consequência da atualidade do tema compreende-se que haja mais publicações nos anos mais recentes. Apesar do ano de 2019 ter apresentado apenas 3 publicações, não há evidências concretas que essa queda tenha sido refletida em toda a comunidade 
acadêmica, pelo contrário, justifica-se pelo fato deste mapeamento ter se iniciado no terceiro trimestre de 2019 e não englobar publicações do último trimestre do ano. A busca pelos estudos primários considerou apenas os 10 últimos anos de publicações, sendo estes estudos encontrados entre 2010 e 2019. Os anos de 2015 e 2016 não apresentaram publicações. $\mathrm{O}$ ano com o maior número de publicações entre os estudos primários foi em 2018, com 7 estudos.

Os EPs foram classificados de acordo com o nível de ensino. As evidências encontradas concentram-se em Ensino Superior e Ensino Técnico. A maioria dos estudos estavam concentrados no nível superior, com representação em 20 estudos primários, o qual corresponde a $90,9 \%$ do total. 1 estudo primário $(4,5 \%)$ relatou uma experiência realizada no ensino técnico de nível médio, e outra $(4,5 \%)$ relatou um estudo de caso tanto na graduação quanto no ensino técnico. Tais resultados sugerem que a Aprendizagem baseada em problemas aplicada ao ensino de Programação tem sido mais difundida no ensino superior, o que justifica-se pelo fato da Programação ser mais difundida na graduação.

Finalmente, no que diz respeito ao país de publicação, os EPs estão distribuídos em 12 países, dos quais o Brasil tem a maior representatividade, com 5 estudos selecionados. Em seguida, Estados Unidos que tiveram 4 estudos, e China, Canadá e Turquia, cada um com 2 estudos. Os outros países não representam um número significativo.

Desse modo, o presente estudo contribuiu para a estruturação sistemática das informações sobre um conjunto de contribuições e desafios da Aprendizagem baseada em Problemas no ensino de Programação, servindo como base para o desenvolvimento de trabalhos futuros na área, bem como para o desenvolvimento de planos de projeto de ABP para o ensino de Programação. Como trabalhos futuros sugere-se refazer esse estudo dentro de 5 anos visando observar se houve alguma mudança nas tendências levantadas no presente texto.

\section{Referências}

DIAS, S. R., CHAGA, M. M. Aprendizagem Baseada em Problema: Um relato de experiência. In: MACHADO A. B. (Org.). Práticas inovadoras em metodologias ativas. Florianópolis: Contexto Digital, 2017. Disponível em https://www.saojose.br/wp-content/uploads/2018/09/praticas_inovadoras_em_metodolo gias_ativas.pdf. Acesso em 13 mai. 2019.

KITCHENHAM, B.; CHARTERS, S.. Guidelines for performing Systematic Literature Reviews in Software Engineering. Keele University. Durham, UK, 2007. Disponível

em: http://citeseerx.ist.psu.edu/viewdoc/download;jsessionid=F4CC8FFAB6D866167E8D6 A43EE7DE61F?doi=10.1.1.117.471\&rep=rep1\&type=pdf. Acesso em 13 mai. 2019.

MOREIRA, G. L. et. al.. Desafios na aprendizagem de Programação introdutória em cursos de TI da UFERSA, campus Pau dos Ferros: um estudo exploratório. In: ECOP, Pau dos Ferros, RN, 2018. Disponível em: https://periodicos.ufersa.edu.br/index.php/ecop. Acesso em 22 mai. 2019.

RAABE, A. L. A., SILVA, J. M. C. Um Ambiente para Atendimento às Dificuldades de Aprendizagem de Algoritmos. In: XXV Congresso da Sociedade Brasileira de Computação. UNISINOS, São Leopoldo, RS. 2005. Disponível em: http://www.lbd.dcc.ufmg.br/colecoes/ wei/2005/003.pdf. Acesso em: 18 mai. 2019. 
RIBEIRO, L. R. C. A Aprendizagem Baseada em problemas (PBL): uma implementação na educação em engenharia na voz dos atores. 2005. Tese de Doutorado em Educação, Universidade Federal de São Carlos, São Carlos. Disponível em:

https://repositorio.ufscar.br/bitstream/handle/ufscar/2353/TeseLRCR.pdf?sequence=1\&i sAll. Acesso em 18 mai. 2019. 